\title{
COMUNICAÇÃO DO DIAGNÓSTICO: IMPLICAÇÕES NO TRATAMENTO DE ADOLESCENTES DOENTES CRÔNICOS
}

\author{
Viviane Ziebell de Oliveira \\ Manoela Ziebell de Oliveira\# \\ William Barbosa Gomes \\ Carolina Gasperin
}

\begin{abstract}
RESUMO. O estudo descreve, interpreta e discute aspectos comunicativos presentes na relação médico-paciente no atendimento de adolescentes portadores de doenças orgânicas crônicas. Foram entrevistados 15 médicos (generalistas e especialistas), 18 adolescentes portadores de doenças crônicas (13 com fibrose cística e cinco com outras doenças), 16 mães, e dois pais. As entrevistas semi-estruturadas foram gravadas, transcritas e analisadas segundo os três passos característicos da análise fenomenológica: descrição qualitativa, indução lógica e interpretação. Os resultados discutem as dificuldades da comunicação de más notícias e as implicações de equívocos nestes procedimentos que podem vir a prejudicar a adesão ao tratamento. As conclusões ressaltam o papel do psicólogo na equipe de saúde, oportunizando melhores condições para o desenvolvimento psicológico destes pacientes, auxiliando na comunicação médico-paciente e favorecendo a adesão ao tratamento.
\end{abstract}

Palavras-chave: Relação médico-paciente, doença orgânica crônica, adolescência.

\section{COMMUNICATION OF THE DIAGNOSTIC: IMPLICATIONS ON THREATENING ADOLESCENTS WITH CHRONIC ILLNESS}

\begin{abstract}
This study describes, interprets and discusses communicative aspects of physician-patient relations in the treatment of adolescents with chronic organic illnesses. Fifteen physicians (generalists and specialists), 18 adolescents (13 with cystic fibrosis and five with other chronic organic diseases), 16 mothers and two fathers, were interviewed. Semistructured interviews were recorded, transcribed and analyzed according to three characteristic phenomenological steps: qualitative description, logical induction and interpretation. The results discuss the difficulties in communicating bad news and the implications of mistakes in such procedures, which may endanger the adhesion to treatment. The conclusions highlight the psychologist part in the health team, offering better conditions to the psychological development of these patients, helping in physician-patient communication and co-operating on treatment adhesion.
\end{abstract}

Key words: physician-patient relation, chronic organic disease, adolescence.

Vários aspectos vêm servindo como indicadores de adesão ao tratamento médico em jovens portadores de doenças crônicas. São eles: percepção dos pais, características peculiares de cada faixa etária, comportamentos relacionados à doença, características do paciente, regime de tratamento, problemas psicossociais do desenvolvimento e relação médicopaciente (Lecussán, 2001; Mulhern, Wasserman, Friedman \& Faiclough, 1989). A utilização da relação médico-paciente como parte do tratamento é tão antiga quanto a Medicina. No passado, quando a tecnologia ainda era pouco desenvolvida, a comunicação verbal era indispensável ao diagnóstico (Tähkä, 1986). No entanto, para alguns pesquisadores (Tay-Yap \& AlHawamdeh, 2001), os objetivos da tecnologia são, justamente, complementar a comunicação médicopaciente e facilitar o acesso à informação. Tais afirmações contrastam com entendimentos anteriores

\footnotetext{
* Doutora em Psicologia pela Universidade Federal do Rio Grande do Sul-UFRGS e Psicóloga do Hospital de Clínicas de Porto Alegre.

\# Bolsista de Iniciação Científica/CNPq do Instituto de Psicologia da UFRGS.

II Doutor em Pisologia, Docente no Instituto de Psicologia da UFRGS.

æ Psicóloga formada pelo Instituto de Psicologia da UFRGS.
} 
nos quais a tecnologia era descrita como que colocando o lado humano do tratamento em segundo plano (Kaplan \& Sadock, 1993) ou preterindo a habilidade de comunicar-se (Foley, 1993).

O objetivo deste estudo é examinar as repercussões da comunicação do diagnóstico, em casos de doenças orgânicas crônicas, na perspectiva de pacientes adolescentes, pais e médicos. $\mathrm{O}$ aspecto focal do estudo é o início da relação médico-paciente, através da informação do diagnóstico, ou seja, a comunicação de más notícias. Desta relação dependerá, em grande parte, a adesão ou não ao tratamento, influenciando o seu sucesso ou fracasso. Por adesão entende-se a colaboração ativa entre o paciente e o seu médico tendo em vista o sucesso terapêutico (Miller, 1997).

\section{MÉTODO}

\section{Informantes}

Participaram como informantes da pesquisa adolescentes portadores de doenças orgânicas crônicas, familiares de adolescentes em atendimento e médicos de várias especialidades que trabalham em um hospital universitário.

O primeiro grupo foi constituído de 18 adolescentes portadores de doenças orgânicas crônicas desde a infância, cujas idades variavam entre 12 e 18 anos, sendo 10 rapazes e oito moças, todos pacientes de um hospital universitário. Dentre os jovens entrevistados, 13 eram portadores de fibrose cística e cinco possuíam outras doenças, como: asma, artrite reumatóide, mielomeningocele (com complicações renais e deficiência física associadas) e "síndrome de imunodeficiência não adquirida" (envolvendo doença reumática e doença renal, em grau severo). Os pacientes participantes da pesquisa estavam em avaliação ou atendimento durante o período delimitado para a pesquisa (oito meses), sendo previamente classificados quanto à boa ou má adesão ao tratamento médico apresentada . O critério de classificação baseava-se na correspondência entre a informação prestada pelo médico e a prestada pelo próprio paciente sobre: comparecimento às consultas, uso de medicações prescritas, mudanças no estilo de vida e cumprimento de dietas. Apenas nove destes pacientes apresentavam sinais de boa adesão.

O segundo grupo constituiu-se de familiares acompanhantes de pacientes adolescentes e de 18 participantes. Destes familiares, dois eram pais e 16 mães, uma das quais era mãe adotiva. O critério de seleção do acompanhante foi a sua participação no momento da comunicação do diagnóstico de doença orgânica crônica dos filhos. Dentre os informantes, nove eram familiares de pacientes adolescentes portadores de fibrose cística e outros nove eram familiares de pacientes que possuíam diagnósticos como: mielomeningocele, varizes esofágicas, púrpura, artrite reumatóide, epilepsia, leucemia, e mucopolissacaridose tipo 1. Cada grupo foi composto de um pai e oito mães.

O terceiro grupo foi constituído por 15 médicos, sendo seis mulheres e nove homens, especialistas em cinco áreas: um pediatra, três pneumologistas pediátricos, cinco hematologistas, três oncologistas pediátricos e três hebiatras. Quatro médicos eram professores da Faculdade de Medicina, oito eram contratados pelo Hospital e três eram médicos residentes. O tempo de experiência no tratamento de doenças crônicas variou entre três e 25 anos, e estava relacionado com a situação funcional dos médicos: em média, três anos entre residentes, 10 anos entre contratados e 20 anos entre os professores.

\section{Materiais e procedimentos}

Foram utilizados três roteiros de entrevista individual, um para cada grupo de participantes. Conforme recomendações de Patton (1990), o entrevistador era livre para introduzir novas perguntas, quando oportuno, e para ampliar o quadro informativo sobre o tema proposto. No entanto, foram preservadas as questões básicas para permitir a comparação entre as respostas das diversas entrevistas. Para esta exposição, serão abordados apenas os itens referentes à informação/comunicação do diagnóstico. Aos pais perguntou-se: "Como foi feito o diagnóstico?"; Para os adolescentes, as questões foram: "Como e quando ficastes sabendo da doença? Quem te contou? Que idade tinhas? E como entendestes o que te disseram?"; Aos médicos, as perguntas foram as seguintes: "Poderias me falar sobre o período de investigação diagnóstica? Para quem é fornecido o diagnóstico? Como isto é feito? O que tem que ser observado? Quais as reações que tens notado nos pais? E nos adolescentes?". As entrevistas foram gravadas em audioteipe e transcritas literalmente. Os adolescentes portadores de doenças orgânicas crônicas foram entrevistados em situação de internação hospitalar, para tratamento de rotina, ou então, em sistema ambulatorial, quando compareceram para consulta médica, também de rotina. Todos eles estavam com a doença controlada na época da entrevista. Os jovens que estavam hospitalizados responderam à entrevista no próprio quarto, onde permaneciam somente o paciente e o entrevistador. Na situação ambulatorial, 
foram utilizados consultórios, mantendo-se também presentes somente o adolescente e o pesquisador. As entrevistas com os acompanhantes foram realizadas na unidade de internação onde os jovens estavam hospitalizados ou então em consultórios ambulatoriais, quando o acompanhamento médico era de regime externo. As entrevistas com os médicos também foram realizadas no hospital. Nas três situações foram explicados os objetivos da pesquisa e atendidas as exigências de consentimento informado.

\section{Análise dos dados}

As análises qualitativas foram realizadas em três etapas: 1) descrição compreensiva das respostas dos participantes à entrevista, elaborada através da demarcação das falas em unidades temáticas, para que nada do que foi dito fosse deixado de fora nem engrandecido ou diminuído por escolhas do pesquisador (Gomes, 1998); 2) identificação de temas que se apresentam com potencialidade de esclarecer os elos que interligam as experiências vividas pelos entrevistados, de acordo com os elementos fornecidos pelo contexto da descrição, designada por Patton (1990) de análise indutiva; 3 ) análise crítica ou interpretativa dos temas identificados através de confronto com a descrição, a literatura e a experiência do pesquisador com a situação em foco (Lanigan, 1994). As três etapas mencionadas são passos reflexivos imbricados, cada passo contendo os três. Deste modo, a análise apresentada a seguir iniciará com uma síntese compreensiva das respostas obtidas (primeira etapa), demarcada em temas (segunda etapa) que, enquanto escolhas do pesquisador, já se constituem em interpretação (terceira etapa). No entanto, nesta síntese compreensiva preserva-se a perspectiva dos entrevistados. Procura-se manter ao longo da análise o enfoque qualitativo, isto é, exclui-se qualquer apoio quantitativo de falas ou falantes, mantendo-se coerentes as especificações de possibilidades (Gomes, 1998).

\section{RESULTADOS}

A apresentação da descrição qualitativa das entrevistas intercala as falas dos pais, dos adolescentes e dos médicos sobre a situação de diagnóstico. $\mathrm{Na}$ descrição evitou-se qualquer referência a comparações quantitativas, ressaltando-se a ocorrência de especificação temática como pura possibilidade. As falas dos entrevistados que em geral ilustram estudos qualitativos foram omitidas para privilegiar o conjunto e o contraste entre as perspectivas dos pais, dos jovens pacientes e dos médicos.
Através dos relatos dos pais, será possível identificar uma diferença em relação à percepção do atendimento recebido por pacientes portadores de fibrose cística e pelos portadores de outras doenças. Isto se justifica pela existência de um serviço especializado no atendimento aos pacientes portadores de fibrose cística nesse Hospital, o que ainda não acontece com as demais doenças mencionadas.

\section{O diagnóstico na percepção dos pais}

As mães, em primeiro lugar, tomaram conhecimento da doença do filho em uma variedade de circunstâncias, incluindo o período de gestação (através de exames pré-natais), o nascimento ou os primeiros meses de vida da criança. A agilidade no conhecimento da doença e do encaminhamento para tratamento esteve relacionada, como esperado, à menor ou maior distância de centros médicos desenvolvidos. Houve ainda situações em que a percepção da mãe antecipou-se à evidência dos sintomas. Por exemplo, os bebês portadores de fibrose cística, em geral, já nasceram com algum sintoma, como secreção e ronqueira ao respirar, obstrução intestinal, tosse e sintomas pós-natais, como diarréia, perda de peso, passando a receber atendimento médico. Não obstante, como esta ainda é uma doença pouco conhecida, até mesmo pelos pediatras, geralmente a sua identificação é demorada, o que requer muitas consultas e exames até que, por fim, o paciente seja encaminhado para uma equipe especializada.

A definição do diagnóstico foi uma jornada difícil. Houve episódios de pacientes que passaram por várias etapas diagnósticas, recebendo notícias controversas, devido ao pouco conhecimento das doenças. Em geral, os diagnósticos foram comunicados em momentos críticos: gestação, nascimento, agudização dos sintomas ou frente a procedimentos de urgência. A comunicação do diagnóstico geralmente foi assumida por um médico, que o informou à mãe. $\mathrm{O}$ modo comunicativo variou de acordo com as situações e o estilo pessoal do médico. Alguns médicos explicaram detalhadamente, outros encaminharam o paciente para especialistas, outros não conseguiram concluir o diagnóstico. Ocorreram situações em que os médicos disseram não haver mais nada a fazer pelo paciente. A evolução da doença muitas vezes exigiu diagnósticos complementares mais tardios, que foram comunicados pelo médico na presença dos pacientes já adolescentes. Mesmo assim, as mães mostraram tendência a pensar que os filhos não compreenderam toda a extensão do problema. 
As apreensões dos pais foram atenuadas em situações nas quais ocorreram informações detalhadas da doença e orientação para o tratamento. Em contraste, atendimentos no sistema público de saúde trouxeram problemas de diagnóstico e de comunicação. A comunicação foi prejudicada, segundo os pais, pela despersonalização do paciente e pelo limitado conhecimento de doenças raras nesses serviços. O diagnóstico também trouxe preocupações sobre questões financeiras.

Nem sempre os familiares compreendiam o que estava sendo dito pelo médico. Como esperado, sentiram-se assustados diante de um prognóstico que previa pouco tempo de vida para os filhos, chegando a experimentar sentimentos de desespero e medo. Houve casos em que esses pais saíram chorando pela rua, quebraram coisas, projetaram a culpa em algo ou em alguém, ou então admitiram que, naquela hora, não entenderam o que estava sendo comunicado. Os pais que já conheciam a doença e que já tinham perdido outros filhos em decorrência do mesmo problema disseram que nesse momento "viram a morte" novamente.

As mães salientaram que foi muito difícil receber o diagnóstico. Dentre as reações imediatas descritas por elas, destaca-se a tentativa de negar a realidade. Elas procuraram se convencer de que os médicos estavam enganados, recorrendo, para tanto, a livros de Medicina ou à opinião de familiares médicos. Posteriormente, os sentimentos de tristeza e de culpa prevaleceram, juntamente com manifestações de nãoaceitação da realidade. A assimilação das informações médicas recebidas foi lenta.

Pais e mães tiveram mais dificuldades para entender a real dimensão do problema em situações nas quais o filho parecia "perfeito", sem nenhum defeito físico visível. A aceitação da causa genética foi difícil em pessoas de baixa escolaridade, e nestes casos, a tendência foi responsabilizar o outro cônjuge ou a família de origem pela doença. Aos poucos, foi sendo esclarecida a participação de ambos os pais, possibilitando que a culpa fosse partilhada.

\section{O diagnóstico na percepção dos adolescentes}

Os jovens cresceram convivendo com tratamentos e ouvindo a conversa de pais e médicos sobre a doença. A identificação da doença, pelos médicos, não foi formalmente compartilhada com os pacientes, pois na época eram bebês ou crianças pequenas, mas sim com os pais. Estimaram que conseguiram entender o que realmente acontecia por volta dos oito ou nove anos de idade. Os adolescentes portadores de fibrose cística disseram nunca terem sido oficialmente comunicados sobre o seu diagnóstico, até porque viviam doentes desde o nascimento. Através do convívio com a doença e dos cuidados da mãe, eles foram gradativamente compreendendo o que se passava. Afirmaram que, embora ninguém tenha lhes falado oficialmente sobre a enfermidade, eles perceberam que ocorria algo errado, porque sempre precisaram de hospitalizações, medicações e fisioterapia, apesar dos seus protestos.

Os informantes relataram que a compreensão da doença ocorreu, principalmente, na adolescência. As implicações decorrentes foram as seguintes: conscientização de ser doente, maior conhecimento da doença, descoberta de preconceito, vergonha de ser diferente, percepção de que não podiam fazer tudo que os outros da mesma idade faziam. Por outro lado, constataram que poderiam buscar maior autonomia, já que a adolescência também lhes trazia condições de fazer, sozinhos, alguns procedimentos rotineiros, como a fisioterapia, por exemplo. No entanto, jovens cuja relação com a mãe caracterizava-se por muita dependência não reconheceram nenhuma modificação neste período de vida.

Houve jovens que afirmaram estar mais conscientes da situação e que a idade facilitou a aceitação do tratamento. Em contraste apareceram evidências do sofrimento que surge ante a constatação das sequielas. A percepção das diferenças físicas em relação aos jovens sadios também foi relacionada à entrada na adolescência. A importância da doença, em alguns casos, foi negada, independentemente da evidência (como na mielomeningocele, onde existe uma deficiência física), ou não (como na asma).O conhecimento da doença foi uma particularidade que diferenciou os pacientes portadores de fibrose cística dos demais entrevistados: eles sabiam que se tratava de doença genética e incurável e descreveramna em detalhes. Os pacientes portadores de outras doenças, por sua vez, descreveram brevemente os sintomas, mas não demonstraram clareza a respeito do seu quadro. Demonstraram ainda dificuldades em se colocar a respeito da doença, procurando ignorar a comunicação manifestada pelo corpo através de sintomas como pressão alta, insuficiência renal, deficiência física. Assim, enquanto a doença se manteve estável, pouco evidente, ou não exigiu intervenções mais invasivas, não foi percebida como grave; mas admitir ser doente mostrou-se sinônimo de "ser anormal".

\section{O diagnóstico na percepção dos médicos}

A comunicação do diagnóstico é em si paradoxal. Trata-se de uma má notícia que deve ser simultânea a uma boa notícia: a doença é incurável, mas pode ser 
tratada. Os entrevistados mostraram-se preocupados com fantasias contraditórias - morte versus cura - que aparecem nestes momentos. Assim, o problema da comunicação deste diagnóstico levanta as seguintes questões: o que deve ser dito? Quem deve dizer? Como deve ser dito? Para quem deve ser dito? Uma preocupação manifestada pelos professores entrevistados refere-se a "quem deve ser o emissário da notícia". Eles têm como norma que a comunicação do diagnóstico deve ser uma tarefa do chefe da equipe que atende o paciente, podendo ser compartilhada com os outros membros, em menor grau.

Os entrevistados divergem sobre o que, como e para quem revelar o diagnóstico; no entanto, são unânimes em reconhecer a importância de passar esperanças para o paciente e sua família. Os médicos revelaram intenção de compartilhar a verdade com os adultos e, ao mesmo tempo, proteger os adolescentes. A rotina estabelecida por regras médicas gerais para a comunicação de diagnósticos aos pais indica que os profissionais devem falar sobre a doença, prognóstico e tratamento, e depois "abrir para perguntas". As informações devem ser fornecidas gradualmente, levando em conta o "tempo" do ouvinte, no caso, a mãe. Nesse momento é necessário que fiquem atentos para as reações do interlocutor, acompanhando-as e deixando espaço para a expressão da tristeza. Para tanto, a sensibilidade e a experiência são fundamentais.

Os entrevistados, no entanto, divergiram em alguns pontos. Ocorreram questionamentos sobre o efeito real de uma atitude mais cautelosa e disponível perante situações que são realmente adversas, já que nestas condições talvez não faça diferença a forma de comunicar a má notícia. Esta posição estava associada à impossibilidade de oferecer a cura ao paciente. Outra posição ressaltava a importância de o médico ter em mente algumas alternativas para administrar o impacto da má notícia. As alternativas apresentadas foram as seguintes: 1) ter um plano de tratamento já definido no momento da comunicação do diagnóstico, como uma forma de não deixar o paciente e a família ao desamparo e o médico sem ter o que fazer pelo tratamento; 2) oferecer a oportunidade de uma nova consulta para poucos dias depois; e 3) sugerir a internação do paciente para trabalhar melhor as reações à notícia. No caso de internação, os médicos contam com o contexto hospitalar formal e informal para ajudar os pais a elaborarem a nova situação. Os informantes consideraram que, para a equipe, é importante observar mais intensamente a mãe, a fim de avaliar suas condições para assumir os cuidados necessários ao bom andamento do tratamento.
A responsabilidade pelo tratamento de pacientes muito jovens é sempre dos pais, o que requer do médico a comunicação com intermediários. A esses intermediários, de acordo com as regras médicas, deve-se assegurar, desde o início da investigação, que qualquer enfermidade, quando identificada, deve ser tratada. A disponibilidade necessária para trabalhar as resistências oferecidas tanto pelos pais quanto pelos pacientes também faz parte das regras médicas básicas. A notícia do diagnóstico deve ser dada para os pais conjuntamente.

\section{A comunicação do diagnóstico aos adolescentes}

A comunicação do diagnóstico ao paciente adolescente mostrou ser a tarefa mais difícil para os médicos entrevistados. As entrevistas apontaram critérios para orientar a comunicação com o adolescente, por exemplo, idade do paciente, vontade dos pais e impasses éticos implícitos. Nos casos de impasse, têm-se dilemas como: pode-se passar toda informação, independentemente da idade ou da vontade dos pais?

Os médicos mostraram-se sensibilizados pelo contato com doenças graves na adolescência, enfatizando as sérias implicações para o desenvolvimento psicológico dos jovens. Disseram que esses pacientes não têm capacidade de ver a extensão do problema, o que se refletirá no tratamento. Por esta razão, fornecer diagnóstico é uma experiência difícil, levando alguns profissionais a evitarem a situação, conversando com o jovem na presença dos paisou apenas com os pais. A idade do médico e a maturidade profissional contribuem para o manejo da situação. Os mais jovens, especialmente os residentes, indicaram dificuldades pela proximidade etária; no entanto, os mais velhos também se referiram a uma outra proximidade, por exemplo, dos jovens com os próprios filhos e sobrinhos.

Os profissionais entendem e defendem que o doente deva conhecer a sua enfermidade através de uma comunicação médica. No entanto, há médicos que nunca passaram pela experiência de informar o diagnóstico ao adolescente, pois, no caso deles, o fato ocorreu tempos atrás, quando o paciente ainda era um bebê. Com efeito, as conversas com a mãe sobre a doença vêm ocorrendo há anos na presença dos pacientes, mas os informantes acreditam que eles não compreendem o que está se passando. Ademais, não estava claro para os médicos se há necessidade e qual o momento de começar a envolver esses pacientes na consulta e falar diretamente sobre o caso. Houve profissionais que assumiram certa reserva sobre a necessidade de o paciente adolescente saber de toda a 
verdade sobre a sua condição. Isto mostra que, embora tenha sido consenso que os pais devem ser informados de tudo, o mesmo não aconteceu com respeito aos adolescentes.

Por fim, os médicos sentem intensamente a dificuldade de levar uma notícia ruim ao jovem, mas enfatizaram que nestas circunstâncias a principal mensagem deve ser de solidariedade.

\section{As reações dos adolescentes}

Os médicos disseram que os jovens, em geral, reagem ao diagnóstico com susto e frieza, comportando-se como se não fossem eles os doentes. Tal postura surpreende, pois esperavam que os adolescentes apresentassem alguma curiosidade em saber sobre a doença. Estes pacientes resistem ao tratamento, alegando medo da dor e inconformidade com as limitações impostas. Os médicos compreendem a resistência como uma característica da própria fase, mas, por outro lado, preocupam-se com a sua dificuldade em enfrentar a realidade da doença.

A partir desta compreensão, as percepções e experiências dos entrevistados dividem-se entre aqueles que evitam relacionar-se diretamente com os adolescentes e aqueles que admitem não saber como lidar com esses pacientes. Os primeiros tendem a manter indefinidamente a mãe como intermediária no tratamento e os outros procuram aprender com os próprios pacientes. No contexto da entrevista, aparece com clareza a necessidade dos médicos de contar com mais recursos técnicos para lidar com essa população. Eles sabem que os jovens pacientes podem prestar informações mais exatas sobre seus sintomas e problemas, e reconhecem que a proximidade auxiliará na preparação dos jovens para assumirem os cuidados necessários.

\section{Reflexões dos médicos sobre a experiência com doenças crônicas}

A experiência foi valorizada como fator de modificação e adaptação de condutas médicas, ou seja, como um espaço que possibilita reflexões e escolhas. Para os entrevistados, o tempo tem mostrado que eles não podem ser evasivos e esquivos. Ao contrário, devem ser claros e honestos, podendo inclusive assumir que não sabem tudo. A experiência ensinou a lidar melhor com as fantasias dos pacientes e com a tendência dos pais de projetarem a culpa do seu infortúnio na equipe de saúde. Disseram, ainda, que o tempo e a experiência são os principais agentes de transformações, e que o papel do médico é de intermediação entre a doença, o paciente e a família. Com o passar dos anos, sentem-se gradativamente mais seguros e empáticos, podendo aceitar melhor que, em algumas situações, os pais não suportem a verdade. Nestes casos, cabe ao médico auxiliar esses pais no enfrentamento dessa realidade.

Os médicos entrevistados acrescentaram às adversidades inerentes à sua profissão a realidade atual da saúde no país, que não lhes permite contar com a estrutura necessária para o atendimento dos pacientes (ambulância, exames, leitos hospitalares, medicações). Colocaram que, neste sistema, os crônicos sempre serão os últimos pacientes atendidos, pois são os que gastam mais, em todos os sentidos, inclusive quanto à atenção médica, e também são os que apresentam retorno menor, em todos os sentidos.

\section{DISCUSSÃO E CONCLUSÕES}

O presente estudo é, basicamente, uma descrição contextual de aspectos comunicativos na relação entre médicos, pacientes adolescentes portadores de doenças orgânicas crônicas, pais e mães de pacientes. $\mathrm{O}$ interesse central foi a comunicação do diagnóstico. A combinação do que foi dito pelos entrevistados com o que não foi claramente expressado (mas sugerido nas entrelinhas) sustentou a compreensão de uma situação real, aqui interpretada como possibilidade e não como veracidade.

Os relatos dos adolescentes mostraram que o contexto existencial da doença orgânica crônica grave é paradoxal, pois está ligado tanto ao amor quanto à morte. A descoberta da doença combinou atos de amor e a presença constante da possibilidade de morte. Entre essas duas condições, os participantes têm de enfrentar a realidade da limitação do tempo de vida e da vigência constante da doença. Para adiar a morte, muito amor e dedicação são exigidos dos cuidadores (pais e equipe de saúde). Diante deste quadro, os profissionais médicos enfrentam a impossibilidade de responder ao objetivo de cura da Medicina, por maior dedicação que venham ter. As mães, por sua vez, acabam abrindo mão de suas vidas pessoais, por amor aos filhos. No entanto, constatou-se que, apesar de cuidados e amados, estes adolescentes dificilmente elaboram projetos de vida. Portanto, a interrogação que fica ausente, mas pode ser depreendida dos depoimentos dos participantes da pesquisa é: "vale a pena investir, se o sofrimento e a morte não serão vencidos?".

A fala dos médicos também sugere dificuldades práticas em lidar com as implicações psicológicas, existenciais e comunicacionais que envolvem as doenças crônicas e a morte. A formação médica, com os avanços instrumentais e farmacológicos, privilegia 
a tecnologia às humanidades, não dando a necessária atenção à construção de habilidades comunicativas.

A revisão pós-coleta de dados do presente estudo mostrou avanços na definição de critérios empíricos que devem ser implementados na relação médicopaciente (Buckman, 2001; Kurtz, 2002; Young, Dixon-Woods, Windridge \& Heney 2003). Nos últimos 20 ou 30 anos avanços científicos e tecnológicos na área da Medicina determinaram a necessidade de intenso treinamento dos profissionais, com a finalidade de assimilar uma enorme quantidade de novos e complexos conhecimentos. Mesmo sendo considerada uma parte da "arte da Medicina", a comunicação médico-paciente continua sendo aprendida somente através da experiência. Por muitos anos, os médicos ficaram expostos ao desconforto gerado pelos sentimentos de inadequação desencadeados pelo enfrentamento de situações mais estressantes. As barreiras na comunicação médicopaciente quase sempre não são explícitas, podendo manifestar-se por meio de mensagens não-verbais, dissonância cognitiva, resistência inesperada, e até mesmo através de sentimentos de desconforto do médico. Para resolvê-las são sugeridos modelos de comunicação baseados na empatia, na exploração de informações e sentimentos e na legitimação; porém, sempre que conflitos forem identificados, as diferenças e os interesses comuns devem ser explicitados (Quill, 1989).

Uma das situações consideradas mais difíceis na relação médico-paciente é a comunicação de más notícias (Morgan \& Winter, 1996; Novack, Plumer, Smith, Ochitill, Morrow \& Bennett, 1979). A preocupação com a comunicação de más notícias foi manifestada desde a criação do primeiro código de ética médica dos Estados Unidos, em 1847 (Girgis \& Sanson-Fischer, 1995), e até os dias de hoje o seu conteúdo e a sua forma são discutidos. Atualmente, a comunicação é considerada um instrumento fundamental para o relacionamento entre médico e paciente e para o alcance das metas terapêuticas, cujos resultados psicológicos, comportamentais e somáticos favoráveis para o paciente são freqüentemente avaliados através de pesquisas (Buckman, 2001; Kurtz, 2002; Hargie, Dickson, Boohan \& Hughes, 1998).

Com o gradativo domínio da tecnologia e em decorrência da nova realidade emergente dos seus resultados, a questão humana foi retomada. Tem sido reconhecido que toda doença leva a um sentimento de isolamento, e todas as pessoas desejam ter os seus sentimentos, idéias e dilemas entendidos por outras pessoas. A comunicação empática passou a ser novamente valorizada. Ela é definida pelos autores como uma habilidade cognitiva, que é passível de ser ensinada e aprendida e envolve a compreensão dos sentimentos do paciente (Platt \& Keller, 1994). Por isto, a sensibilidade do médico voltou a ser considerada um recurso importante, juntamente com o uso de técnicas de comunicação (Garg, Buckman \& Kason, 1997).

Esta revalorização de uma postura médica mais humana tem exigido um investimento na formação acadêmica. A preocupação com o ensino da comunicação médico-paciente tem sido manifestada por escolas de Medicina do mundo inteiro. Novos programas destinados a ensinar estudantes de medicina a fornecer más notícias a pacientes e familiares empática e consistentemente são elaborados e testados freqüentemente, como comprova a literatura (Quill, 1989; Morgan \& Winter, 1996; Novack, Plumer, Smith, Ochitill, Morrow \& Bennett, 1979; Hargie, Dickson, Boohan \& Hughes, 1998; Platt \& Keller, 1994; Garg, Buckman \& Kason, 1997; Novack, Dubé \& Goldstein, 1992; Roter, Rosenbaum, Negri, Renaud, DiPrete-Brown \& Hernandez, 1998). As pesquisas mostram que o treinamento ajuda os médicos a falarem menos e escutarem mais os seus pacientes. Os resultados ainda englobam a maior utilização de perguntas abertas, facilitadoras, e identificam uma fala médica emocionalmente mais responsiva. Os pacientes destes médicos, em contrapartida, passaram a falar mais positivamente e apresentaram uma tendência a fornecer-lhes mais informações nas consultas (Roter, Rosenbaum, Negri, Renaud, DiPrete-Brown \& Hernandez, 1998).

Apesar do grande investimento no ensino de habilidades comunicacionais nos cursos de Medicina, constata-se uma preocupação ainda unidirecional e tecnicista. Unidirecional porque vai do médico para o paciente, e tecnicista porque tem ênfase em regras informativas. A preocupação com o enfrentamento e com o manejo dos próprios sentimentos tem sido pouco contemplada na formação médica (Morgan \& Winter, 1996); porém, comunicação não é sinônimo de informação. A informação implica em um contexto de escolha dado, cabendo ao sujeito aceitá-lo e obedecer a ele. A comunicação, por sua vez, permite ao sujeito utilizar a sua própria experiência para fazer julgamentos a respeito da informação, e só então escolher o que faz sentido para ele, em cada situação. Toda comunicação, portanto, necessita de uma informação, mas não basta uma informação para haver uma comunicação. Na comunicação o sujeito parte da idéia para a experiência, fazendo uso da abstração 
para resolver os diferentes problemas que se the apresentam (Lanigan, 1994).

O papel da equipe de saúde é fundamental no tratamento de crianças e adolescentes portadores de doenças orgânicas crônicas, como ficou evidenciado, neste estudo, no contraste entre $\mathrm{o}$ atendimento aos pacientes portadores de fibrose cística e aos portadores de outras doenças crônicas. Reitera-se que o médico é o principal responsável pelo tratamento do paciente e ocupa uma posição de autoridade que é fundamental em todo o processo. $\mathrm{O}$ médico foi quem acolheu a mãe e orientou-a na realização do tratamento, possibilitando a sobrevida do paciente.

A comunicação do diagnóstico não é função do psicólogo hospitalar. No caso de doenças orgânicas crônicas, cabe a esse profissional acompanhar $\mathrm{o}$ tratamento desde os passos iniciais e auxiliar no processo normal de desenvolvimento dos pacientes adolescentes, bem como na construção de uma relação positiva entre a família, o paciente e a equipe de saúde. A discussão de aspectos relacionais e comunicativos junto à equipe de saúde, a grupos de mães/pais e de pacientes é uma possibilidade importante a ser explorada pelo psicólogo. Quanto às mães/pais, cabe ajudá-los a diferenciarem o indivíduo (filho) da doença, processo difícil, porque a comunicação fica centrada apenas nos procedimentos que envolvem o tratamento.

A participação cada vez maior do psicólogo nas instituições hospitalares vem exigindo uma literatura própria, que atenda às especificidades desta área de pesquisa e de prática profissional. Em certos casos, a perspectiva psicológica aponta para dificuldades relacionais não facilmente admitidas e muitas vezes tratadas como tabu, em particular, a relação médicopaciente. Em princípio, a qualidade da relação médico-paciente é uma responsabilidade médica e, por conseguinte, discutida e orientada em programas de formação médica; contudo, em determinadas situações, como no atendimento de doenças orgânicas crônicas, a qualidade desta relação é a condição fundamental para a adesão ao tratamento médico. Abre-se, então, a possibilidade da intervenção do psicólogo no sentido de facilitar esta comunicação.

\section{REFERÊNCIAS}

Buckman, R. (2001). Communication skills in palliative care. Neurologic Clinics, 19 (4), 989-1004.

Foley, G.B. (1993). Enhancing child-family-health team communication. Cancer, 71(10), 3281-3289.

Garg, A, Buckman, R. \& Kason, Y. (1997). Teaching medical students how to break bad news. Can. Med. Assoc. J., 156(8), 1159-1164.
Girgis, A. \& Sanson-Fisher, R.W. (1995). Breaking bad news: consensus guidelines for medical practitioners. Journal of Clinical Oncology, 13, 2449-2456.

Gomes, W.B. (1998). Apresentação: fenomenologia e pesquisa em psicologia. Em W.B. Gomes (Org.) Fenomenologia e pesquisa em psicologia. Porto Alegre: Editora da Universidade.

Hargie, O., Dickson, D., Boohan, M. \& Hughes, K. (1998). A survey of communication skills training in UK schools of medicine: present practices and prospective proposals. Medical Education, 32, 25-34.

Kaplan, H. \& Sadock, (6 ${ }^{\mathrm{a}}$.ed) (1993). Compêndio de psiquiatria: ciências comportamentais. Porto Alegre: Artes Médicas.

Kurtz, S.M. (2002). Doctor-patient communication: principles and practices. Canadian Journal of Neurological Sciences, 29(Suppl2): S23-S29.

Lanigan, R.L. (1994). Capta versus data: method and evidence in communicology. Human Studies, 17, 109-130.

Lecussán, P. (2001). A criança e o diagnóstico: o pediatra deve contar à criança doente seu diagnóstico? Revista da Associação Médica Brasileira, 47 (4), 282-283.

Miller, N.H. (1997). Compliance with treatment regimens in chronic asymptomatic diseases. The American Journal of Medicine. 102 (2A), 43-48.

Morgan, E.R. \& Winter, R.J. (1996). Teaching communication skills. Arch. Pediatr. Adolesc. Med., 150, 638-641.

Mulhern, R.K., Wasserman, A.L., Friedman, A.G. \& Faiclough, D. (1989). Social competence and behavioral adjustment of children who are long-term survivors of cancer. Pediatrics, 83 (1), 18-25.

Novack, D.H., Plumer, R., Smith, R.L., Ochitill, H., Morow, G.R. \& Bennett, R.M. (1979). Changes in physicians attitudes toward telling the cancer patient. JAMA, 241(9), 897-899.

Novack, D.H., Dubé, C. \& Goldstein, M.G. (1992). Teaching medical interviewing. A basic course on interviewing and physician-patient relationship. Arch. Intern. Med., 152, 1814-1820.

Patton, M. (1990). Qualitative evaluation methods. Beverly Hills, Ca.: Sage Publications, Inc.

Platt, F.W. \& Keller, V.F. (1994). Empathic communication: A teachable and learnable skill. Journal of General Internal Medicine, 9, 222-226.

Quill, T. E. (1989). Recognizing and adjusting to barriers in doctor-patient communication. Annals of Internal Medicine, 111(1), 51-57.

Roter, D., Rosenbaum, J., Negri, B., Renaud, D., DiPreteBrown, L. \& Hernandez, O (1998). The effects of a continuing medical education programme in interpersonal communication skills on doctor practice and patient satisfaction in Trinidad and Tobago. Medical Education, 32, 181-189.

Tähkä, V. (1986). O relacionamento médico-paciente. Porto Alegre: Artes Médicas.

Tay-Yap, J. \& Al-Hawamdeh, S. (2001). The impact of the Internet on healthcare in Singapore. Journal-of-ComputerMediated-Communication, 6 (4), (fascículo não-paginado). 
Young, B., Dixon-Woods, M., Windridge, K.C. \& Heney D. (2003). Managing communication with young people who have a potentially life threatening chronic illness: qualitative study of patients and parents. British Medical Journal, 326

Recebido em 20/06/2003

(7384), 305-308.

Aceito em 30/11/2004

Endereço para correspondência: Viviane Ziebell de Oliveira, Rua São Luiz, 1101, CEP 90620-170, Porto Alegre-RS. E-mail: viziebell@terra.com.br 